\title{
UNIQUENESS IN CAUCHY PROBLEMS FOR HYPERBOLIC DIFFERENTIAL OPERATORS
}

\author{
CHRISTOPHER D. SOGGE
}

\begin{abstract}
In this paper we prove a unique continuation theorem for second order strictly hyperbolic differential operators. Results also hold for higher order operators if the hyperbolic cones are strictly convex. These results are proved via certain Carleman inequalities. Unlike [6], the parametrices involved only have real phase functions, but they also have Gaussian factors. We estimate the parametrices and associated remainders using sharp $L^{p}$ estimates for Fourier integral operators which are due to Brenner [1] and Seeger, Stein, and the author [5].
\end{abstract}

\section{INTRODUCTION}

The main purpose of this work is to establish local unique continuation theorems for second order (strictly) hyperbolic operators with smooth coefficients. Thus, we shall work with hyperbolic differential operators

$$
P(x, D)=\sum_{j, k=1}^{n} g^{j k}(x) D_{j} D_{k}+\sum_{j=1}^{n} b_{j}(x) D_{j}
$$

whose coefficients are in $C^{\infty}(X)$, where $X$ is a connected open subset of $\mathbf{R}^{n}$ with $n \geq 3$. As usual $D=i^{-1}\left(\partial / \partial x_{1}, \ldots, \partial / \partial x_{n}\right)$. At the end of the paper we shall also consider the case where $n=2$, and we shall also prove a uniqueness theorem for higher order hyperbolic differential operators which involves a convexity assumption on the characteristic varieties of the principal symbol.

In the elliptic case we showed [6] that if

$$
|P(x, D) u| \leq|V u|,
$$

where $V \in L_{\mathrm{loc}}^{n / 2}(X)$, then $u$ must vanish identically if it vanishes in a nonempty open subset of $X$, which generalized earlier results of Jerison and Kenig [3]. It is well known that nothing as strong can hold in the hyperbolic setting. We shall show that there is always unique continuation across "space-like" surfaces to solutions of (1.2) involving potentials $V$ as in the elliptic unique continuation theorem.

To state this result more precisely, we first need to recall the definition of the normal set $N(F) \subset T^{*} X \backslash 0$ of a closed subset $F \subset X . N(F)$ is the set of all

Received by the editors October 30, 1989 and, in revised form, August 6, 1990.

1991 Mathematics Subject Classification. Primary 35B45, 35B60.

The author was supported in part by a Sloan Fellowship and the NSF. 
$\left(x_{0}, \xi_{0}\right)$, where $x_{0} \in F, 0 \neq \xi_{0} \in \mathbf{R}^{n}$, and

$$
\Psi(x) \leq \Psi\left(x_{0}\right) \text { when } x_{0} \in F,
$$

for some real $C^{\infty}$ function satisfying $d \Psi\left(x_{0}\right)= \pm \xi_{0}$.

In addition, we must define the notion of "time-like" directions. Let

$$
p(x, \xi)=\sum_{j, k=1}^{n} g^{j k}(x) \xi_{j} \xi_{k}
$$

denote the principal symbol of $P(x, D)$. Then, following [2, Chapter 28], we shall let

$$
\begin{array}{r}
\Sigma=\left\{(x, N) \in T^{*} X \backslash 0: p(x, \xi+\tau N) \text { has a double root } \tau\right. \\
\text { with } \left.\xi+\tau N \neq 0 \text { for some } \xi \in \mathbf{R}^{n}\right\} .
\end{array}
$$

It is easy to see that $\Sigma$ is closed and conic. In the case of the usual constant coefficient wave operator on $\mathbf{R}^{n}, \Sigma$ would just be the set of all $(x, N)$, where $N$ does not belong to the (open) forward or backward light cone. Therefore, we shall call $(x, N) \in T^{*} X \backslash 0$ a time-like vector if $(x, N) \notin \Sigma$. Also a smooth surface is called space-like if all of its normal vectors are time-like.

We can finally state our main result.

Theorem 1.1. Suppose that $D^{\alpha} u \in L_{\text {loc }}^{2}(X),|\alpha| \leq 1$, and that $u$ satisfies the differential inequality (1.2) with $V \in L_{\mathrm{loc}}^{n / 2}(X)$, where $n \geq 3$ is the dimension. It then follows that $N(\operatorname{supp} u) \subset \Sigma$, where $\Sigma$ is defined by (1.3).

Thus there is always unique continuation across space-like surfaces. Another consequence is that there is uniqueness in the Cauchy problem involving $P(x, D)+V$, when the initial surface is space-like.

In the special case where the potential is locally bounded, this result is a special case of the Calderón uniqueness theorem (see [2, Vol. IV, p. 221]), and in fact one can assume that the coefficients are merely Lipschitz of order one. On the other hand, little seems to be known for unbounded potentials. The only previous work seems to be due to Kenig, Ruiz, and the author [4]. In this paper a unique continuation theorem was proved for constant coefficient operators when both $V$ and $u$ satisfy certain global integrability conditions. On the other hand, if these global assumptions are fulfilled, it was shown that there is unique continuation across any hyperplane. In addition, there was a unique continuation theorem involving only local integrability assumptions on $u$ and $V$, but one had to assume that the differential inequality (1.2) held in a light cone, rather than a relatively compact set.

Let us now discuss why the unique continuation theorem for all hyperplanes cannot be true in the present local setting. The counterexamples involve the constant coefficient wave operator $P(D)=D_{1}^{2}-\sum_{j=2}^{n} D_{j}^{2}$. First of all, a classical example (see [2, Theorem 8.6.7]) shows that one can have $C^{\infty}$ solutions to $P(D) u=0$ whose support is a half-space with characteristic boundary. Furthermore, an example of P. Cohen (see [2, Theorem 13.6.1]) shows that there is an $a(x) \in C^{\infty}$ so that $[P(D)+\langle a(x), \nabla\rangle] u=0$ has a solution having support equal to a noncharacteristic half-space whose normal is not time-like. In other words, there is not unique continuation across noncharacteristic surfaces that are not space-like. To summarize, the conclusion that $N(\operatorname{supp} u) \subset \Sigma$ cannot be replaced by a stronger one where $\Sigma$ is replaced by a smaller set. 
To prove Theorem 1.1 we must show that there is local unique continuation across any space-like surface $S$. That is, if $u$ satisfies (1.2) and if $u$ vanishes in an open set below $S$, then we must show that $u$ vanishes in a neighborhood of $S$. By making a change of variables (see [2, Vol. III, pp. 500-502]) we can assume that $S=\left\{x: x_{1}=\left|x^{\prime}\right|^{2}, x^{\prime}=\left(x_{2}, \ldots, x_{n}\right)\right\}$ and that

$$
P(x, D)=D_{1}^{2}-\sum_{j, k>1} g^{j k}(x) D_{j} D_{k}+\sum b_{j}(x) D_{j},
$$

where now $\left(g^{j k}(x)\right)$ is an $(n-1)$ by $(n-1)$ positive definite matrix depending smoothly on $x$. We must show that if supp $u \cap\left\{x: x_{1} \geq 0\right\}=\{0\}$, and if $P(x, D)$ is now as in $\left(1.1^{\prime}\right)$, then $u$ vanishes in a neighborhood of 0 if it satisfies (1.2). But standard arguments (see [2, Chapter 17]) imply that this follows from the following Carleman inequalities.

Theorem 1.2. Let $P(x, D)$ be as in $\left(1.1^{\prime}\right)$ and put

$$
w=x_{1}-x_{1}^{2} \text {. }
$$

Then if $\lambda>0$ is sufficiently large

$$
\left\|e^{-\lambda w} v\right\|_{L^{p^{\prime}}} \leq C\left\|e^{-\lambda w} P v\right\|_{L^{p}}
$$

whenever $v \in C_{0}^{\infty}$ is supported in a small neighborhood $\mathcal{N}$ of 0 . Here $p=$ $2 n /(n+2)$ and $p^{\prime}$ is the conjugate exponent $p^{\prime}=2 n /(n-2)$, which means that $1 / p-1 / p^{\prime}=2 / n$.

These Carleman estimates are analogous to the ones in the elliptic case [6]. However, when $P(x, D)$ is elliptic, $\sum_{|\alpha| \leq 1} \lambda^{1+1 / n-|\alpha|}\left\|e^{-\lambda w} D^{\alpha} u\right\|_{L^{p}}$ is also controlled by the right-hand side of (1.5) (if $g^{j k}$ is close to a constant matrix). On the other hand, for hyperbolic operators it seems doubtful that one can have the same estimates for $D^{\alpha} u,|\alpha|=1$, and $p$ as above. This explains why we are assuming that the lower order terms in (1.1) are smooth.

If one replaces $v$ by $e^{-\lambda w} v$ in (1.5), then one sees that it suffices to make estimates for the conjugated operators

$$
P_{\lambda}=e^{-\lambda w} P e^{\lambda w}
$$

The proof of these estimates will involve two main steps. First one constructs a parametrix for $P_{\lambda}$ by adapting the arguments in [6] for the elliptic case. The parametrix is a Fourier integral operator with singular symbol whose kernel is (microlocally) of the form

$$
(2 \pi)^{-n} e^{-\lambda\left(x_{1}-y_{1}\right)^{2}} \int_{\mathbf{R}^{n}} \frac{\alpha e^{i \Phi}}{p\left(x, \xi-i \lambda w^{\prime}\right)} d \xi .
$$

The phase function $\Phi$ is real and parametrizes the trivial canonical relation, while $\alpha$ is a standard (compound) symbol of order zero. One constructs $\Phi$ from an eikonal equation involving $\xi_{1} \pm \sqrt{\sum_{j, k>1} g^{j k}(x) \xi_{j} \xi_{k}}$, and then $\alpha$ is constructed from a transport equation which has the same form as the one occurring in the construction of parametrices for the Cauchy problem. Having constructed the parametrix, the next step involves estimating the $L^{p} \rightarrow L^{p^{\prime}}$ and $L^{p^{\prime}} \rightarrow L^{p^{\prime}}$ mapping properties of the parametrix and the associated remainder. 
In the arguments for the elliptic and parabolic case [6, 7] this involved techniques that were first used for the restriction theorem and spherical summation theorems in Fourier analysis. In the present setting, though, we shall be able to argue more directly, using estimates for nondegenerate Fourier integral operators. The $L^{p} \rightarrow L^{p^{\prime}}$ estimates we shall use are due to Brenner [1], while the $L^{p^{\prime}} \rightarrow L^{p^{\prime}}$ estimates are recent results of Seeger, Stein, and the author [5].

During the preparation of this article we learned that B. Barcelo and A. Ruiz have announced similar results for second order operators.

It is a pleasure to thank A. Seeger and E. M. Stein. Our collaboration greatly influenced this work.

\section{PARAmetrix CONSTRUCtion}

Let $P_{\lambda}$ be as in (1.6). Then the desired inequality (1.5) is clearly equivalent to

$$
\|v\|_{L^{p^{\prime}}} \leq C\left\|P_{\lambda} v\right\|_{L^{p}}, \quad \operatorname{supp} v \subset \mathcal{N}, \lambda>0 \text { large. }
$$

To prove this inequality we intend to construct operators $T=T_{\lambda}$ and $R=R_{\lambda}$ satisfying the equation

$$
T P_{\lambda}=\text { Identity }+R
$$

and the estimates

$$
\begin{aligned}
\|T\|_{\left(L^{p}, L^{p^{\prime}}\right)} & \leq C, \\
\|R\|_{\left(L^{p^{\prime}}, L^{p^{\prime}}\right)} & \leq C \lambda^{-1 / n},
\end{aligned}
$$

when acting on functions supported in $\mathscr{N}$. Clearly, (2.2)-(2.4) yield (2.1) when $\lambda>0$ is large.

Notice that the symbol of $P_{\lambda}$ is

$$
P_{\lambda}(x, \xi)=p\left(x, \xi-i \lambda w^{\prime}\right)+\sum b_{j}(x) \xi_{j}-i b_{1}(x) \lambda w^{\prime}-\lambda w^{\prime \prime} .
$$

As before, $p(x, \xi)$ is the principal symbol of $P$. The last two terms in the right-hand side of $(2.5)$ are junk terms and will only contribute to the remainder. Unlike $[6,7]$, the first-order term $\sum b_{j}(x) \xi_{j}$ is important, and it will play a role in the transport equations to follow.

The first step is to notice that it is easy to invert $P_{\lambda}$ in certain regions of $T^{*} X$. To be more specific, let $\Gamma$ denote the "light cones":

$$
\Gamma=\left\{(x, \xi) \in T^{*} X: p(x, \xi)=0\right\} .
$$

We then let $T_{0}$ be the integral operator with kernel

$$
(2 \pi)^{-n} \int \eta_{\lambda}(x, \xi) \frac{e^{i\langle x-y, \xi\rangle}}{p\left(x, \xi-i \lambda w^{\prime}\right)} d \xi,
$$

where $\eta_{\lambda}$ is a symbol of order zero having the following properties. First

$$
\operatorname{supp} \eta_{\lambda} \subset\{(x, \xi):|\xi| \leq c \lambda\} \cup T^{*} X \backslash \widetilde{\Gamma},
$$

where $\widetilde{\Gamma}$ is a small conic neighborhood of $\Gamma$, and $c>0$ is a small constant. In addition, we assume that

$$
\eta_{\lambda}(\xi)=1 \quad \text { if }|\xi| \leq c \lambda / 2 \text { or } \xi \in T^{*} X \backslash \widetilde{\widetilde{\Gamma}}
$$


where $\widetilde{\widetilde{\Gamma}} \subset \widetilde{\Gamma}$ is a smaller conic neighborhood of $\Gamma$. The reason we have chosen $\eta_{\lambda}$ as above is that

$$
\left|p\left(x, \xi-i \lambda w^{\prime}\right)\right| \geq c_{0}\left(|\xi|^{2}+\lambda^{2}\right), \quad(x, \xi) \in \operatorname{supp} \eta_{\lambda}, \text { some } c_{0}>0,
$$

provided that $\tilde{\widetilde{\Gamma}}$ and $c$ above are small enough. Thus, inverting $P_{\lambda}$ on $\operatorname{supp} \eta_{\lambda}$ is an elliptic problem, and in fact straightforward arguments using the pseudodifferential calculus show that, if $T_{0}$ is as above, then $T_{0}$ satisfies $(2.3)$, and

$$
T_{0} P_{\lambda} v=(2 \pi)^{-n} \iint e^{i\langle x-y, \xi\rangle} \eta_{\lambda}(x, \xi) v(y) d \xi d y+R_{0} v
$$

for a remainder term satisfying estimates which are better than we require:

$$
\left\|R_{0}\right\|_{\left(L^{p^{\prime}}, L^{p^{\prime}}\right)} \leq C \lambda^{-1}
$$

For similar arguments see [6].

On account of this we must construct operators $T_{1}$ and $R_{1}$ satisfying estimates as in (2.3)-(2.4) and the equation

$$
T_{1} P_{\lambda} v=(2 \pi)^{-n} \iint e^{i\langle x-y, \xi\rangle} \rho_{\lambda}(x, \xi) v(y) d \xi d y+R_{1} v
$$

with

$$
\rho_{\lambda}(x, \xi)=1-\eta_{\lambda}(x, \xi) .
$$

$T_{1}$ will be an integral operator having a kernel of the form

$$
(2 \pi)^{-n} e^{-\lambda\left(x_{1}-y_{1}\right)^{2}} \int \rho_{\lambda}(x, \xi) \frac{\alpha(x, y, \xi) e^{i \Phi(x, y, \xi)}}{p\left(x, \xi-i \lambda w^{\prime}\right)} d \xi .
$$

The phase function $\Phi$ will be constructed from eikonal equations similar to those occurring in parametrix constructions for the Cauchy problem for $P$. $\Phi$ will be real and it will parameterize the trivial relation, i.e.,

$$
\partial \Phi / \partial \xi=0 \Leftrightarrow x=y \quad \text { and } \quad \partial \Phi / \partial x=-\partial \Phi / \partial y=\xi \quad \text { when } x=y .
$$

Thus, we shall require that

$$
\Phi=\langle x-y, \xi\rangle+O\left(|x-y|^{2}|\xi|\right) .
$$

Once $\Phi$ is chosen, $\alpha$ will be constructed from a transport equation involving $\Phi$ that is identical to the ones in Cauchy problems. The Gaussian factor in (2.7) will play a crucial role in the estimates for $T_{1}$ and especially $R_{1}$. As we shall see, it occurs because of the concavity of the Carleman weights $w$.

To construct $T_{1}$ we shall first construct a right parametrix $T_{1}^{*}$ for the adjoint of $P_{\lambda}$. To motivate this construction notice that if we let $\Phi_{\lambda}(x, y, \xi)=$ $\Phi(x, y, \xi)+i \lambda\left(x_{1}-y_{1}\right)^{2}$, where $\Phi$ is an unknown phase function, and if $Q(x, D)$ equals $P^{*}(x, D)$ minus the zero order term, then

$$
\begin{gathered}
P_{\lambda}^{*}(x, D)\left[\alpha(x, y, \xi) e^{i \Phi_{\lambda}(x, y, \xi)}\right]=\alpha p\left(x, \partial \Phi_{\lambda} / \partial x+i \lambda w^{\prime}\right) \\
-i\left\{\sum_{j, k=1}^{n} g^{j k}(x) \frac{\partial \alpha}{\partial x_{j}} \frac{\partial \Phi}{\partial x_{k}}-\alpha Q(x, D) \Phi\right\}+\text { junk },
\end{gathered}
$$


where, if $\alpha$ is a symbol of order zero, the junk terms have the property that, after dividing by $\lambda$, they are in a bounded subset of symbols of order zero. We shall first construct $\Phi$ so that

$$
\frac{p\left(x, \partial \Phi_{\lambda} / \partial x+i \lambda w^{\prime}\right)}{p\left(x, \xi+i \lambda w^{\prime}\right)}
$$

is a symbol of order zero which equals one along the diagonal. Thus, the numerator cancels out the singularity coming from the denominator. Next, after $\Phi$ is chosen, we shall solve a transport equation so that the term inside the braces in (2.9) vanishes. We shall also require that $\alpha=1$ along the diagonal. Doing all of this will provide us with a right parametrix whose error term has favorable bounds, and we can then get (2.7) by taking adjoints.

Phase function construction. The first step is to notice that we have the factorization

$$
p\left(x, \xi+i \lambda w^{\prime}\right)=\left(\xi_{1}-q\left(x, \xi^{\prime}\right)+i \lambda w^{\prime}\right)\left(\xi_{1}+q\left(x, \xi^{\prime}\right)+i \lambda w^{\prime}\right) .
$$

Here $q$ is the first-order symbol which is elliptic in $\xi^{\prime}=\left(\xi_{2}, \ldots, \xi_{n}\right)$ and is given by

$$
q\left(x, \xi^{\prime}\right)=\sqrt{\sum_{j, k>1} g^{j k}(x) \xi_{j} \xi_{k}} .
$$

Based on this factorization, we see that it might be well to construct $\Phi$ differently in the two regions $\operatorname{supp} \rho_{\lambda} \cap\left\{\xi: \xi_{1}>0\right\}$ and $\operatorname{supp} \rho_{\lambda} \cap\left\{\xi: \xi_{1}<0\right\}$.

If we are in the forward region, where $\xi_{1}>0$, then the first factor is the nonelliptic one. Hence, as we just pointed out, we should try to find $\Phi_{\lambda}$ so that

$$
\partial \Phi_{\lambda} / \partial x_{1}-q\left(x, \partial \Phi_{\lambda} / \partial x^{\prime}\right)+i \lambda w^{\prime}(x)=\xi_{1}-q\left(y, \xi^{\prime}\right)+i \lambda w^{\prime}(y) .
$$

As we shall see, we can find a phase function $\Phi(x, y, \xi)$ satisfying (2.10) on $\operatorname{supp} \rho_{\lambda}$ so that, if we put

$$
\Phi_{\lambda}=\Phi+i \lambda\left(x_{1}-y_{1}\right)^{2},
$$

then $(2.11)$ holds.

The first step in constructing $\Phi$ is to "freeze" $x_{1}=y_{1}$ and then find a phase function $\phi$ involving one less variable satisfying

$$
\begin{gathered}
q\left(y_{1}, x^{\prime}, \partial \phi / \partial x^{\prime}\right)=q\left(y, \xi^{\prime}\right), \\
\phi=\left\langle x^{\prime}-y^{\prime}, \xi^{\prime}\right\rangle+O\left(\left|x^{\prime}-y^{\prime}\right|^{2}\left|\xi^{\prime}\right|\right) .
\end{gathered}
$$

To do this we recall that (for $x^{\prime}$ close to $y^{\prime}$ ) there is a unique phase function $\phi$ satisfying (2.13) together with the initial condition that $\phi=0$ when $\left\langle x^{\prime}-y^{\prime}, \xi^{\prime}\right\rangle=0$, and $\partial \phi / \partial x^{\prime}=\xi^{\prime}$ when $x^{\prime}=y^{\prime}$. This condition clearly implies the other requirement (2.14).

Having constructed $\phi$ we would like to find a phase function $\psi(x, y, \xi)$ satisfying

$$
\psi=\left(x_{1}-y_{1}\right) \xi_{1}+O\left(\left|x_{1}-y_{1}\right|^{2} \xi_{1}\right) \quad \text { on } \operatorname{supp} \rho_{\lambda} \cap\left\{\xi: \xi_{1}>0\right\},
$$

so that if we take $\Phi=\phi+\psi$, then

$$
\partial \Phi / \partial x_{1}-q\left(x, \partial \Phi / \partial x^{\prime}\right)=\xi_{1}-q\left(y, \xi^{\prime}\right) .
$$


Once again, there is a unique solution $\psi$ to $\left(2.11^{\prime}\right)$ near the diagonal if, this time, we impose the initial condition that $\psi=0$ when $x_{n}=y_{n}$ and $\partial \psi / \partial x=$ $\left(\xi_{1}, 0, \ldots, 0\right)$ when $x=y$. Since $\xi_{1} \approx\left|\xi^{\prime}\right|$ on $\operatorname{supp} \rho_{\lambda} \cap\left\{\xi: \xi_{1}>0\right\}$, one easily checks that $\psi$ is as in (2.15).

Since $\Phi$ clearly satisfies (2.10), and since one sees that $\Phi_{\lambda}$ in (2.12) satisfies (2.11), this concludes the construction in the region where $\xi_{1}>0$.

To construct $\Phi_{\lambda}$ in the other region, we use the other factor in (2.10). Thus, we wish to have $\Phi_{\lambda}$ as above, except we require that it solves the equation

$$
\partial \Phi_{\lambda} / \partial x_{1}+q\left(x, \partial \Phi_{\lambda} / \partial x^{\prime}\right)+i \lambda w^{\prime}(x)=\xi_{1}+q\left(y, \xi^{\prime}\right)+i \lambda w^{\prime}(y) .
$$

To do this we use the same phase function $\phi$ as above, but we require that $\psi$ satisfy the analogues of $(2.15)$ and $\left(2.11^{\prime}\right)$ in the backward region, where in $\left(2.11^{\prime}\right)$ the minuses are replaced by pluses. If one then lets $\Phi_{\lambda}=\phi+\psi+$ $i \lambda\left(x_{1}-y_{1}\right)^{2}$, then $\Phi_{\lambda}$ has the desired properties on supp $\rho_{\lambda} \cap\left\{\xi: \xi_{1}<0\right\}$.

Transport equation. Now that $\Phi$ has been chosen we would like to find $\alpha$ so that the second term in (2.9) is zero:

$$
\sum_{j, k=1}^{n} g^{j k}(x) \frac{\partial \Phi}{\partial x_{j}} \frac{\partial \alpha}{\partial x_{k}}=\alpha Q(x, D) \Phi .
$$

Also, we shall need that $\alpha$ is of order zero and

$$
\alpha(x, y, \xi)=1 \quad \text { when } x=y .
$$

Notice that $(2.16)$ is a linear differential equation whose coefficients are homogeneous of degree one in $\xi$. Thus, since $\sum g^{j k}(x) \frac{\partial \Phi}{\partial x_{j}} \frac{\partial}{\partial x_{k}}$ is a nondegenerate real vector field and since $g^{11} \equiv 1$, we conclude that there is a unique $\alpha$ which is homogeneous of degree zero in $\xi$ satisfying (2.16) and the condition that $\alpha=1$ when $x_{1}=y_{1}$, which is stronger than (2.17).

Having chosen $\Phi$ and $\alpha$, let us now compute $P_{\lambda}^{*}(x, D) T_{1}^{*}$. The kernel of this operator equals $(2 \pi)^{-n}$ times

$$
\begin{aligned}
P^{*}(x, D) & {\left[e^{-\lambda\left(x_{1}-y_{1}\right)^{2}} \int \rho_{\lambda}(y, \xi) \frac{\alpha e^{i \Phi}}{p\left(y, \xi+i \lambda w^{\prime}\right)} d \xi\right] } \\
= & e^{-\lambda\left(x_{1}-y_{1}\right)^{2}} \int \rho_{\lambda}(y, \xi) \frac{\alpha p\left(x, \partial \Phi / \partial x+i \lambda w^{\prime}\right)}{p\left(y, \xi+i \lambda w^{\prime}\right)} e^{i \Phi} d \xi \\
& +e^{-\lambda\left(x_{1}-y_{1}\right)^{2}} \int \rho_{\lambda}(y, \xi) \frac{\alpha_{\lambda} e^{i \Phi}}{p\left(y, \xi+i \lambda w^{\prime}\right)} d \xi .
\end{aligned}
$$

The symbol $\alpha_{\lambda}$ can be computed explicitly. By (2.16), it must be

$$
\alpha_{\lambda}=\left[2 \lambda+\sum D_{j} b_{j}(x)\right] \alpha+2 \lambda\left(x_{1}-y_{1}\right) \partial \alpha / \partial x_{1},
$$

since $\sum D_{j} b_{j}(x)$ is the zero order term of $P^{*}(x, D)$. Thus, as we said before, $\lambda^{-1} \alpha_{\lambda}$ is in a bounded subset of symbols of order zero.

Next, we claim that the first term in the right-hand side of (2.18) equals the adjoint of the first term in the right-hand side of (2.6) plus a negligible error. To see this we recall that $\Phi$ and $\alpha$ were chosen so that

$$
\rho_{\lambda}(x, \xi) \frac{\alpha p\left(x, \partial \Phi_{\lambda} / \partial x+i \lambda w^{\prime}\right)}{p\left(y, \xi+i \lambda w^{\prime}\right)}
$$


is a symbol of order zero equaling $\rho_{\lambda}(y, \xi)$ along the diagonal. Consequently, since $\rho_{\lambda}(y, \xi)$ vanishes for $|\xi| \leq c \lambda$, one can use $(2.8)$ and a variation on the equivalence of the phase function theorem for pseudo-differential operators to see that the term under consideration equals

$$
(2 \pi)^{-n} \int e^{i\langle x-y, \xi\rangle} \rho_{\lambda}(y, \xi) d \xi+R_{1,0}^{*},
$$

where $R_{1,0}$ sends $L^{p^{\prime}} \rightarrow L^{p^{\prime}}$ with norm $O\left(\lambda^{-1}\right)$.

Putting all of this together we see from taking adjoints that

$$
T_{1} P_{\lambda} v=(2 \pi)^{-1} \iint e^{i\langle x-y, \xi\rangle} \rho_{\lambda}(x, \xi) v(y) d \xi d y+R_{1,0} v+R_{1,1} v
$$

where $R_{1,1}$ is the adjoint of the integral operator whose kernel is the last term in (2.18). We have just seen that $R_{1,0}$ satisfies better estimates than are needed, so we shall be done if we can estimate $T_{1}$ and $R_{1,1}$.

Let us abuse notation a bit and write

$$
\begin{aligned}
T_{1} v & =(2 \pi)^{-n} e^{-\lambda\left(x_{1}-y_{1}\right)^{2}} \iint \rho_{\lambda}(x, \xi) \frac{\alpha e^{i \Phi(x, y, \xi)}}{p\left(x, \xi-i \lambda w^{\prime}\right)} v(y) d \xi d y \\
R_{1,1} v & =(2 \pi)^{-n} e^{-\lambda\left(x_{1}-y_{1}\right)^{2}} \iint \rho_{\lambda}(x, \xi) \frac{\alpha_{\lambda} e^{i \Phi(x, y, \xi)}}{p\left(x, \xi-i \lambda w^{\prime}\right)} v(y) d \xi d y
\end{aligned}
$$

Since we have taken adjoints the new phase functions should be the old ones with the roles of $x$ and $y$ switched, and similarly $\alpha$ and $\alpha_{\lambda}$ here are the complex conjugates of the old symbols with $x$ and $y$ interchanged. The important thing is that $\Phi$ still satisfies (2.8) and the symbols have the same size. Notice that the main remainder $R_{1,1}$ behaves essentially like $\lambda T_{1}$.

The next section will be devoted to proving the missing inequalities

$$
\begin{aligned}
\left\|T_{1}\right\|_{\left(L^{p}, L^{p^{\prime}}\right)} & \leq C, \\
\left\|R_{1,1}\right\|_{\left(L^{p^{\prime}}, L^{p^{\prime}}\right)} & \leq C \lambda^{-1 / n} .
\end{aligned}
$$

As before, it is understood that the norms are over $\mathscr{N}$ involving functions supported in this set, where $\mathscr{N}$ is a small neighborhood of the origin.

\section{ESTIMATING THE PARAMETRIX}

To prove (2.22)-(2.23) we need to use some sharp estimates for nondegenerate Fourier integral operators having canonical relations which are close to the trivial one. The $L^{r} \rightarrow L^{r^{\prime}}$ estimates are due to Brenner [1], while the $L^{r} \rightarrow L^{r}$ ones are due to Seeger, Stein, and the author [5].

Lemma 3.1. Let $q(x, t, \xi) \in C^{\infty}\left(\mathbf{R}^{d} \times \mathbf{R} \times \mathbf{R}^{d} \backslash 0\right), d \geq 2$, be real and homogeneous of degree one in $\xi$. If $\phi(x, y, \xi)$ is a phase function satisfying

$$
\phi=\langle x-y, \xi\rangle+O\left(|x-y|^{2}|\xi|\right),
$$

and if $a$ is a symbol of order zero, then for $1<r<\infty$

$$
\begin{aligned}
& \| \int_{\mathbf{R}^{d}} \int_{\mathbf{R}^{d}} e^{i[\phi(x, y, \xi)+t q(x, t, \xi)]} a(x, y, \xi) \\
& \quad \times(1+t|\xi|)^{-(d-1)|1 / r-1 / 2|} f(y) d \xi d y\left\|_{L^{r}\left(\mathbf{R}^{d}\right)} \leq C\right\| f \|_{L^{r}\left(\mathbf{R}^{d}\right)},
\end{aligned}
$$


provided that $t>0$ is small and the symbol vanishes when $|x|+|y|$ is larger than a fixed constant. If, in addition,

$$
\operatorname{rank}\left(\partial^{2} q / \partial \xi_{j} \partial \xi_{k}\right) \equiv d-1,
$$

then for $1 \leq r \leq 2,1 / r+1 / r^{\prime}=1$,

$$
\begin{aligned}
\| \int_{\mathbf{R}^{d}} \int_{\mathbf{R}^{d}} e^{i[\phi(x, y, \xi)+t q(x, t, \xi)]} a(x, y, \xi) & \\
& \times|\xi|^{-(d+1)|1 / r-1 / 2|} f(y) d \xi d y\left\|_{L^{\prime}\left(\mathbf{R}^{d}\right)} \leq C t^{-(d-1)(1 / r-1 / 2)}\right\| f \|_{L^{\prime}\left(\mathbf{R}^{d}\right)} .
\end{aligned}
$$

To prove (2.22) and (2.23) we shall now use an argument of Strichartz [8]. Let us start by estimating $T_{1}$. We have to handle the forward region $\left\{\xi: \xi_{1}>\right.$ $0\}$ and the backward region $\left\{\xi: \xi_{1}<0\right\}$ differently. So let $\rho_{\lambda}^{+}$denote the restriction of $\rho_{\lambda}(x, \xi)$ to the forward region, and $T_{1}^{+}$the associated operator. Since $\rho_{\lambda}(x, \xi)$ was chosen to vanish for $|\xi| \leq c \lambda$ and $\xi$ outside of a small conic neighborhood of the light cones, it is clear that $\rho_{\lambda}^{+}$is still a symbol of order zero. Using (2.10) we see that the kernel of $T_{1}^{+}$is

$$
(2 \pi)^{-n} e^{-\lambda\left(x_{1}-y_{1}\right)^{2}} \int \rho_{\lambda}^{+}(x, \xi) \frac{\alpha(x, y, \xi) e^{i \Phi(x, y, \xi)}}{\left(\xi_{1}-q\left(x, \xi^{\prime}\right)-i \lambda w^{\prime}\right)\left(\xi_{1}+q\left(x, \xi^{\prime}\right)-i \lambda w^{\prime}\right)} d \xi .
$$

Let us fix $x_{1}$ and $y_{1}$ in this kernel, and consider the $L^{p}\left(\mathbf{R}^{n-1}\right) \rightarrow L^{p^{\prime}}\left(\mathbf{R}^{n-1}\right)$ operator norm of the resulting integral operator, which we denote by $\widetilde{T}_{1}^{+}$. Recall that $\Phi=\phi+\psi$, where $\phi$ is a phase function on $\mathbf{R}^{n-1}$ satisfying the hypotheses of Lemma 3.1 and $\psi$ satisfies

$$
\psi=\left(x_{1}-y_{1}\right) \xi_{1}+O\left(\left|x_{1}-y_{1}\right|^{2}\left|\xi_{1}\right|\right)
$$

on supp $\rho_{\lambda}$. In particular, if $|x|+|y|$ is small enough, $\partial \psi / \partial \xi_{1}=0 \Leftrightarrow x_{1}=y_{1}$. Using this fact, one sees that

$$
\begin{aligned}
& (2 \pi)^{-n} \int_{-\infty}^{\infty} \rho_{\lambda}^{+}(x, \xi) \frac{\alpha(x, y, \xi) e^{i \psi\left(x, y, \xi^{\prime}\right)}}{\left(\xi_{1}-q\left(x, \xi^{\prime}\right)-i \lambda w^{\prime}\right)\left(\xi_{1}+q\left(x, \xi^{\prime}\right)-i \lambda w^{\prime}\right)} d \xi_{1} \\
& \quad=\frac{a\left(x, y, \xi^{\prime}\right)}{\left|\xi^{\prime}\right|} e^{i\left(x_{1}-y_{1}\right) q\left(x, \xi^{\prime}\right)},
\end{aligned}
$$

where $a$ is a symbol of order zero vanishing for $\left|\xi^{\prime}\right| \leq c \lambda$, some $c>0$. Thus, if as before $x^{\prime}=\left(x_{2}, \ldots, x_{n}\right)$, we have

$$
\left(\widetilde{T}_{1}^{+} g\right)\left(x^{\prime}\right)=e^{-\lambda\left(x_{1}-y_{1}\right)^{2}} \int_{\mathbf{R}^{n-1}} e^{i\left[\phi\left(x^{\prime}, y^{\prime}, \xi^{\prime}\right)+\left(x_{1}-y_{1}\right) q\left(x, \xi^{\prime}\right)\right]} a\left(x, y, \xi^{\prime}\right)\left|\xi^{\prime}\right|^{-1} g\left(y^{\prime}\right) d y^{\prime} .
$$

We can apply inequality (3.2) for $d=n-1$ to estimate this operator since $(d+1)(1 / p-1 / 2)=1$, if $p$ is as in equation (2.22), and since $\operatorname{rank}\left(\partial q / \partial \xi_{j} \partial \xi_{k}\right) \equiv n-2$. The estimate we obtain is

$$
\left\|\widetilde{T}_{1}^{+} g\right\|_{L^{p^{\prime}\left(\mathbf{R}^{n-1}\right)}} \leq C\left|x_{1}-y_{1}\right|^{-1+2 / n}\|g\|_{L^{p}\left(\mathbf{R}^{n-1}\right)} .
$$

Using this inequality and Minkowski's integral inequality yields the estimate

$$
\begin{aligned}
& \left\|T_{1}^{+} f\right\|_{L^{\prime}\left(\mathbf{R}^{n}\right)} \\
& \quad \leq C\left(\int_{-\infty}^{\infty}\left[\int_{\infty}^{\infty}\left\|f\left(x_{1}-y_{1}, \cdot\right)\right\|_{L^{p}\left(\mathbf{R}^{n-1}\right)}\left|x_{1}-y_{1}\right|^{-1+2 / n} d y_{1}\right]^{p^{\prime}} d x_{1}\right)^{1 / p^{\prime}} .
\end{aligned}
$$


But $1 / p-1 / p^{\prime}=2 / n$, and hence one can use the Hardy-Littlewood-Sobolev inequality to see that the last expression is $\leq C\|f\|_{p}$. Since the same type of argument gives the $L^{p} \rightarrow L^{p^{\prime}}$ boundedness of the backward operator $T_{1}^{-}$, we get (2.22).

Notice that so far we have not used the rapid decay of the kernels in the $\left(x_{1}-y_{1}\right)$ direction. To prove $(2.22)$ we shall need to do this. Recall that $R_{1,1} \approx \lambda T_{1}$. Thus, if $R_{1,1}^{+}$is the forward remainder operator, and if $\widetilde{R}_{1,1}^{+}$is the integral operator obtained from fixing the first variables, we can repeat the above argument, but this time using (3.1), to see that

$$
\begin{aligned}
\| \widetilde{R}_{1,1}^{+} & g \|_{L^{p^{\prime}}\left(\mathbf{R}^{n-1}\right)} \\
& \leq C \lambda e^{-\lambda\left(x_{1}-y_{1}\right)^{2}} \lambda^{-1+(n-2)\left|1 / p^{\prime}-1 / 2\right|}\left|x_{1}-y_{1}\right|^{(n-2)\left|1 / p^{\prime}-1 / 2\right|}\|g\|_{L^{p^{\prime}}\left(\mathbf{R}^{n-1}\right)} \\
& =C \lambda^{1-2 / n} e^{-\lambda\left(x_{1}-y_{1}\right)^{2}}\left|x_{1}-y_{1}\right|^{1-2 / n}\|g\|_{L^{p^{\prime}}\left(\mathbf{R}^{n-1}\right)} .
\end{aligned}
$$

However, since

$$
\lambda^{1-2 / n} \int_{-\infty}^{\infty} e^{-\lambda x_{1}^{2}}\left|x_{1}\right|^{1-2 / n} d x_{1}=\text { const } \cdot \lambda^{-1 / n}
$$

one can use Young's inequality and the last estimate to get

$$
\left\|R_{1,1}^{+} f\right\|_{L^{p^{\prime}}\left(\mathbf{R}^{n}\right)} \leq C \lambda^{-1 / n}\|f\|_{L^{p^{\prime}\left(\mathbf{R}^{n}\right)}} .
$$

This finishes the proof, since the same argument proves this estimate for $R_{1,1}^{-}$.

\section{RESULTS IN TWO DIMENSIONS}

Let $P(x, D)$ be a second-order hyperbolic differential operator as in (1.1) in $X \subset \mathbf{R}^{2}$. Then the subset $\Sigma \subset T^{*} X \backslash 0$ defined in (1.3) is very simple. It is just the light cones $\Gamma=\{(x, \xi): p(x, \xi)=0\}$. With this in mind, the analogue of Theorem 1.1 is the following.

Theorem 4.1. Let $u$ satisfy the hypotheses of Theorem 1.1 in a connected open set $X \subset \mathbf{R}^{2}$. Suppose that $|P(x, D) u| \leq V|u|$ in $X$, with $V \in L^{r}(X)$ for some exponent $r>1$. It then follows that $N(\operatorname{supp} u) \subset \Gamma$. Thus there is unique continuation across any noncharacteristic surface.

To prove this result we must show that when $P$ is as in $\left(1.1^{\prime}\right)$,

$$
\left\|e^{-\lambda w} v\right\|_{L^{p^{\prime}}} \leq C\left\|e^{-\lambda w} P v\right\|_{L^{p}}, \quad 1<p \leq 2,
$$

under the same assumptions as in (1.5). It seems unlikely that this inequality can hold for $p=1$, since even the elliptic part of the parametrix for $P_{\lambda}$ cannot be bounded from $L^{1} \rightarrow L^{\infty}$ due to the fact that its kernel has a logarithmic singularity along the diagonal.

Nonetheless it is an easy matter to check that the proof of Theorem 1.2 yields (4.1). One first observes that the elliptic part of the parametrix, $T_{0}$, sends $L^{p} \rightarrow L^{p^{\prime}}$ for any $1<p \leq 2$. This is because $T_{0}$ is a pseudo-differential operator of order -2 . The argument for the nonelliptic part of the parametrix also follows from similar arguments. In the relevant case of $d=1$, Lemma 3.1 
becomes much simpler since it is just a theorem about singular integrals in $\mathbf{R}$. Inequality (3.1) still holds for $d=1$, but (3.2) is only true for $r>1$.

\section{UNIQUENESS THEOREMS FOR HIGHER ORDER OPERATORS}

The purpose of this section is to extend our results to include strictly hyperbolic differential operators. Thus let $P(x, D)$ be a differential operator of order $m$ with smooth coefficients and a real principal symbol. For a given $x$, $P$ is said to be strictly hyperbolic in the direction $N \in \mathbf{R}^{n} \backslash 0$ if the $m$ th order polynomial $\tau \rightarrow p(x, \tau N+\xi)$ has $m$ distinct real roots $\lambda_{j}(\xi)$ for any $\xi \in \mathbf{R}^{n} \backslash 0$ satisfying $\langle\xi, N\rangle=0$. Let $H \subset T^{*} X \backslash 0$ denote the set of strictly hyperbolic directions. Clearly $H$ is open. Our unique continuation theorem will involve the compliment of a smaller open set $H_{c}$. We shall say that $(x, N) \in H$ is in $H_{c}$ if $d_{\xi \xi}^{2} \lambda_{j}(\xi)$ always has rank $n-2$ for any $\xi \in \mathbf{R}^{n} \backslash 0$ with $\langle\xi, N\rangle=0$, where the Hessian is taken in this subspace. Using the change of variables formula for the principal symbol of a differential operator one sees that the condition $(x, N) \in H_{c}$ is well defined since it does not depend on a particular choice of coordinates. If $\Sigma_{c}$ denotes the compliment of the "(strictly) convex hyperbolic directions" $H_{c}$, our result is the following.

Theorem 5.1. Let $P(x, D)$ be as above and suppose that $|P(x, D) u| \leq V|u|$ with $V \in L^{q}(X)$. It then follows that $N(\operatorname{supp} u) \subset \Sigma_{c}$ if $D^{\alpha} u \in L_{\mathrm{loc}}^{2},|\alpha| \leq$ $m-1$, and

(1) $q=n / m$ and $n<m$,

(2) $q>1$ and $m=n$,

(3) $q=1$ and $m>n$.

It is easy to see that this result includes Theorems 1.1 and 4.1. Also, when $m$ is odd the result is vacuous since $\Sigma_{c}=T^{*} X \backslash 0$ in this case. Thus, from now on we shall assume that $m$ is even.

One proves Theorem 5.1 as before by Carleman inequalities. If $\xi_{0}=(1,0$, $\ldots, 0) \in H_{c}$ at $0 \in X$, one can choose local coordinates preserving the direction $\left(0, \xi_{0}\right)$ so that

$$
P(x, D)=D_{x_{1}}^{m}+\sum_{j=1}^{m-1} D_{x_{1}}^{j} P_{j}\left(x, D_{x^{\prime}}\right),
$$

where $P_{j}$ is a differential operator of order $m-j$ acting on $x^{\prime}=\left(x_{2}, \ldots, x_{n}\right)$. Thus in order to prove that $\left(0, \xi_{0}\right) \notin N(\operatorname{supp} u)$, it would be enough to show that, for functions supported in a small neighborhood $\mathscr{N}$ of 0 ,

$$
\left\|e^{-\lambda w} v\right\|_{L^{p^{\prime}}} \leq C\left\|e^{-\lambda w} P v\right\|_{L^{p}}
$$

where everything is as before except now the conjugate exponents satisfy

(1) $1 / p-1 / p^{\prime}=m / n$ if $m<n$,

(2) $1<p \leq 2$ if $m=n$,

(3) $p=1, p^{\prime}=\infty$ if $m>n$.

Let us start with (1) since it is the most difficult. To accomplish this we note that by (5.1) the principal symbol factors as

$$
p(x, \xi)=\left(\xi_{1}-\Lambda_{1}\left(x, \xi^{\prime}\right)\right) \cdots\left(\xi_{1}-\Lambda_{m}\left(x, \xi^{\prime}\right)\right) .
$$


Note that the convexity assumption is just that, for every $j$, the homogeneous functions $\Lambda_{j}$ have Hessians satisfying

$$
\operatorname{rank}\left(\partial^{2} \Lambda / \partial \xi_{j} \partial \xi_{k}\right)_{2 \leq j, k \leq n}=n-2
$$

By (5.3)

$$
p\left(x, \xi+i \lambda w^{\prime}\right)=\left(\xi_{1}-\Lambda_{1}\left(x, \xi^{\prime}\right)+i w^{\prime}\right) \cdots\left(\xi_{1}-\Lambda_{m}\left(x, \xi^{\prime}\right)+i w^{\prime}\right) .
$$

Thus, $\left|p\left(x, \xi+i \lambda w^{\prime}\right)\right| \geq c_{0}\left(|\xi|^{m}+\lambda^{m}\right)$, some $c_{0}>0$, if $|\xi| \leq c \lambda$ for $c>0$ small or if $\xi$ is outside of a small conic neighborhood of

$$
\Gamma=\{(x, \xi): p(x, \xi)=0\}=\bigcup_{j=1}^{m}\left\{(x, \xi): \xi_{1}=\Lambda_{j}\left(x, \xi^{\prime}\right)\right\}
$$

Consequently, if we define $T_{0}$ just as in $\S 2$, we see that standard elliptic arguments imply that $T_{0}: L^{p} \rightarrow L^{p^{\prime}}$, and

$$
T_{0} P_{\lambda}=(2 \pi)^{-n} \iint e^{i\langle x-y, \xi\rangle} \eta_{\lambda}(x, \xi) v(y) d \xi d y+R_{0} v
$$

where this time $R_{0}$ satisfies

$$
\left\|R_{0}\right\|_{\left(L^{p^{\prime}}, L^{p^{\prime}}\right)} \leq C \lambda^{-(m-1)} .
$$

To construct the nonelliptic part of the parametrix, i.e., $T_{1}$, one first chooses the phase function $\Phi$ exactly as before. Thus, if $\rho_{\lambda}(x, \xi)=1-\eta_{\lambda}(x, \xi)$, $\Phi$ has to be chosen differently in each of the $m$ conic neighborhoods of the individual cones in (5.5) making up the support of $\rho_{\lambda}$. Once $\Phi$ is chosen, the zero order symbol $\alpha$ in (2.7) must be chosen. In order to insure that the remainder, $R_{1}$, is a contraction on $L^{p^{\prime}}$, this time it is necessary to recursively solve $m-1$ transport equations. The first equation is exactly the same as the first one occurring in the solution to the Cauchy problem for $P$ (see $[9, \mathrm{pp}$. 310-312]), and the other $m-2$ ones are similar. The resulting symbol $\alpha$ will be a sum of symbols of order $0,-1, \ldots,-(m-2)$ on supp $\rho_{\lambda}$. The symbol of the main remainder will involve $\alpha_{\lambda}$ satisfying this time the following analogue of (2.19):

$$
\alpha_{\lambda}=\lambda \alpha_{\lambda, 0}+\lambda\left(x_{1}-y_{1}\right) \alpha_{\lambda, 1}+\cdots+\left[\lambda\left(x_{1}-y_{1}\right)\right]^{m-1} \alpha_{\lambda, m},
$$

with the $\alpha_{\lambda, j}$ being symbols of order zero.

This describes the parametrix construction for the nonelliptic part of $P_{\lambda}$. Let us now see why the resulting operators $T_{1}$ and $R_{1,1}$ given by (2.20) and (2.21) satisfy the estimates

$$
\begin{aligned}
\left\|T_{1}\right\|_{\left(L^{p}, L^{p^{\prime}}\right)} & \leq C, \\
\left\|R_{1,1}\right\|_{\left(L^{p^{\prime}}, L^{p^{\prime}}\right)} & \leq C \lambda^{-1 / n},
\end{aligned}
$$

if as above $1 / p-1 / p^{\prime}=m / n$.

To prove (5.7) one argues just as before except one uses an easy variant of (3.2):

$$
\begin{aligned}
& \left\|\int_{\mathbf{R}^{n-1}} \int_{\mathbf{R}^{n-1}} e^{i[\phi+t q]} a\left(x, y, \xi^{\prime}\right)\left|\xi^{\prime}\right|^{-(m-1)} f\left(y^{\prime}\right) d \xi^{\prime} d y^{\prime}\right\|_{L^{p^{\prime}\left(\mathbf{R}^{n-1}\right)}} \\
& \quad \leq C t^{-1+m / n}\|f\|_{L^{p}\left(\mathbf{R}^{n-1}\right)}
\end{aligned}
$$


Using this one gets (5.7) by repeating the previous arguments. Notice that this is the place where we need to use the convexity assumption (5.4).

To prove the other inequality, (5.8), one just uses (3.1) in its present form. Let $\widetilde{R}_{1,1}$ be the operator in $\mathbf{R}^{n-1}$ obtained by freezing the first variables in the kernel. Then, since $\left|1 / p^{\prime}-1 / 2\right|=m / 2 n$, (3.1) implies that it has an $L^{p^{\prime}}\left(\mathbf{R}^{n-1}\right) \rightarrow L^{p^{\prime}}\left(\mathbf{R}^{n-1}\right)$ norm which is

$$
\begin{aligned}
\leq & C \lambda e^{-\lambda\left(x_{1}-y_{1}\right)^{2}} \lambda^{-(m-1)+(n-2) \frac{m}{2 n}}\left|x_{1}-y_{1}\right|^{(n-2) \frac{m}{2 n}} \\
& +C \sum_{j=1}^{m-1}\left|\lambda\left(x_{1}-y_{1}\right)\right|^{j} e^{-\lambda\left(x_{1}-y_{1}\right)^{2}} \lambda^{-(m-1)+(n-2) \frac{m}{2 n}}\left|x_{1}-y_{1}\right|^{(n-2) \frac{m}{2 n}} .
\end{aligned}
$$

But

$$
\begin{aligned}
& \lambda^{-m / 2-m / n+2} \int_{-\infty}^{\infty} e^{-\lambda x_{1}^{2}}\left|x_{1}\right|^{m / 2-m / n} d x_{1} \\
& =\text { const } \cdot \lambda^{3 / 2-3 m / 4-m / 2 n} \leq \text { const } \cdot \lambda^{-1 / n},
\end{aligned}
$$

and for $1 \leq j \leq m-1$

$$
\begin{aligned}
& \lambda^{j+1-m / 2-m / n} \int_{-\infty}^{\infty} e^{-\lambda x_{1}^{2}}\left|x_{1}\right|^{j+m / 2-m / n} d x_{1} \\
& \quad=\text { const } \cdot \lambda^{(j+1) / 2-3 m / 4-m / 2 n} \leq \text { const } \cdot \lambda^{-1 / n} .
\end{aligned}
$$

Thus, by arguing as before, we conclude that (5.8) must hold as well.

The proof of the Carleman inequalities for cases (2) and (3) follows from easy modifications of this argument which are similar to those in the previous section.

\section{REFERENCES}

1. P. Brenner, $L_{p}-L_{p^{\prime}}$ estimates for Fourier integral operators related to hyperbolic equations, Math. Z. 152 (1977), 273-286.

2. L. Hörmander, The analysis of linear partial differential operators, Vols. I-IV, SpringerVerlag, New York, Berlin, 1985.

3. D. Jerison and C. E. Kenig, Unique continuation and absence of positive eigenvalues for Schrödinger operators, Ann. of Math. (2) 121 (1985), 463-494.

4. C. E. Kenig, A. Ruiz, and C. D. Sogge, Uniform Sobolev inequalities and unique continuation for second order constant coefficient differential operators, Duke Math. J. 53 (1987), 543-546.

5. A. Seeger, C. D. Sogge, and E. M. Stein, Regularity properties of Fourier integral operators, Ann. of Math. 134 (1991), 231-251.

6. C. D. Sogge, Oscillatory integrals, Carleman inequalities and unique continuation for second order elliptic differential equations, J. Amer. Math. Soc. 2 (1989), 491-516.

7. $\ldots$ A unique continuation theorem for second order parabolic differential operators, Ark. Mat. 28 (1990), 159-182.

8. R. Strichartz, A priori estimates for the wave equation and some applications, J. Funct. Anal. 5 (1970), 218-235.

9. F. Treves, Introduction to pseudodifferential and Fourier integral operators, Vol. II, Plenum Press, New York, 1982. 